\title{
Approximating Stationary Points of Multivalued Generalized Nonexpansive Mappings in Metric Spaces
}

\author{
Kifayat Ullah, ${ }^{1}$ Junaid Ahmad $\mathbb{D}^{1},{ }^{1}$ Muhammad Arshad $\left(\mathbb{D},{ }^{2}\right.$ Manuel de la Sen $\left(\mathbb{D},{ }^{3}\right.$ \\ and Muhammad Safi Ullah Khan ${ }^{1}$ \\ ${ }^{1}$ Department of Mathematics, University of Science and Technology, Bannu, 28100 Khyber Pakhtunkhwa, Pakistan \\ ${ }^{2}$ Department of Mathematics, International Islamic University, H-10, Islamabad 44000, Pakistan \\ ${ }^{3}$ Institute of Research and Development of Processes, University of the Basque Country, Campus of Leioa (Bizkaia), P.O. Box 644, \\ Bilbao, Barrio Sarriena, 48940 Leioa, Spain
}

Correspondence should be addressed to Junaid Ahmad; ahmadjunaid436@gmail.com

Received 12 June 2020; Revised 20 July 2020; Accepted 22 July 2020; Published 17 August 2020

Academic Editor: Zine El Abiddine Fellah

Copyright ( $) 2020$ Kifayat Ullah et al. This is an open access article distributed under the Creative Commons Attribution License, which permits unrestricted use, distribution, and reproduction in any medium, provided the original work is properly cited.

In this research, under some appropriate conditions, we approximate stationary points of multivalued Suzuki mappings through the modified Agarwal-O'Regan-Sahu iteration process in the setting of 2-uniformly convex hyperbolic spaces. We also provide an illustrative numerical example. Our results improve and extend some recently announced results of the current literature.

\section{Introduction}

Let $M=(M, \rho)$ be a metric space and $Y$ be a nonempty subset of $M$. For $a \in M$, set

$$
\begin{gathered}
\rho(a, Y)=\inf \{\rho(a, b): b \in Y\}, \\
\mathscr{R}(a, Y)=\sup \{\rho(a, b): b \in Y\} .
\end{gathered}
$$

Let $\mathscr{C}(Y)$ represent the set of all nonempty compact subsets of $Y$. The function $\mathrm{H}$ defined by

$$
\begin{array}{r}
\mathbb{H}(S, W)=\max \left\{\sup _{u \in S} \rho(u, W), \sup _{v \in W} \rho(v, S)\right\}, \\
\text { for each } S, W \in \mathscr{C}(Y),
\end{array}
$$

satisfies all the properties of the metric and is often called the Hausdorff metric on $\mathscr{C}(Y)$. Recall that a multivalued mapping $F: Y \longrightarrow \mathscr{C}(Y)$ is called the Suzuki mapping [1] if for each $a, b \in Y$,

$$
\frac{1}{2} \rho(a, F a) \leq \rho(a, b) \Rightarrow \mathbb{H}(F a, F b) \leq \rho(a, b) .
$$

The class of Suzuki mappings and its extensions in the setting of single-valued mappings are widely studied by many authors (see, e.g., [2-7] and references therein). Here, we will only focus on the multivalued version of Suzuki mappings. We can easily observe that if $F$ is nonexpansive, that is, $\mathbb{H}(F a, F b) \leq \rho(a, b)$ for all $a, b \in Y$, then $F$ is also a Suzuki mapping. Nevertheless, the following example shows that the converse of this statement may not hold in general.

Example 1. Let $Y=[1,4]$ and $F: Y \longrightarrow \mathscr{C}(Y)$ be defined by

$$
F a= \begin{cases}\{1\}, & \text { for } a \neq 4 \\ {[1.9,2],} & \text { for } a=4\end{cases}
$$

When $a \in(3,4)$ and $b=4$, then we have nothing to prove, because in this case, we have the following:

$$
\begin{aligned}
& \frac{1}{2} \rho(a, F a)=\frac{a-1}{2}>1>\rho(a, b), \\
& \frac{1}{2} \rho(b, F b)=1>\rho(a, b) .
\end{aligned}
$$


When $a<b$ and $(a, b) \in(Y \times Y)-((3,4) \times\{4\})$. In this case, $F$ is nonexpansive, and hence, $F$ is a Suzuki mapping.

By setting $a=3.5$ and $b=4$, we have $\mathbb{H}(F a, F b)=1>$ $0.5=\rho(a, b)$. Hence, $F$ is not nonexpansive.

An element $q$ in $Y$ is called a stationary point (or called an endpoint) of $F$ whenever $\{q\}=F q$ and is called a fixed point of $F$ whenever $q \in F q$. Throughout the paper, the notations $\mathcal{S}(F)$ and $\mathscr{F}(F)$ will represent the set of all stationary points and the set of all fixed points of $F$, respectively. Recall that a multivalued mapping $F: Y \longrightarrow \mathscr{C}(Y)$ is called quasinonexpansive provided that $\mathbb{H}(F a, F q) \leq \rho(a, q)$ for each $a \in Y$ and $q \in \mathscr{F}(F)$. Existence of stationary points for different types of multivalued mappings is studied in [8-15]. The following statements hold:

(i) $\mathcal{S}(F) \subseteq \mathscr{F}(F)$

(ii) $q \in \mathscr{F}(F)$ if and only if $\rho(q, F q)=0$

(iii) $q \in \mathcal{S}(F)$ if and only if $\mathscr{R}(q, F q)=0$

In 2005, Sastry and Babu [16] published a paper on the strong convergence of the fixed point for multivalued nonexpansive mappings using modified Mann and Ishikawa iterative processes in the setting of Hilbert spaces. In the year 2008, Panyanak [17] showed that the results of Sastry and Babu [16] can be extended to the slightly general context of uniformly convex Banach spaces. Song and Wang [18] improved the results of Panyanak [17]. For more details in this direction, see $[19,20]$ and others. In the year 2018, Panyanak [21] published a paper on the approximation of stationary points of multivalued nonexpansive mappings in the framework of Banach spaces using the modified Ishikawa iterative process. In 2019, Ullah et al. [22] quickly noted that the results of Panyanak [21] can be extended to the general context of CAT(0) spaces. In 2020, Laokul and Panyanak [23] used the Ishikawa iterative process for finding stationary points of multivalued Suzuki mappings in 2uniformly convex hyperbolic spaces. Recently, Abdeljawad et al. [24] used the modified Agarwal-O'Regan-Sahu iterative process for finding stationary points of multivalued nonexpansive mappings in Banach spaces. Very recently, Ullah et al. [25] extended the results of Abdeljawad et al. [24] to the general context of 2-uniformly convex hyperbolic spaces. The modified Agarwal-O’Regan-Sahu iteration process reads as follows:

$$
\left\{\begin{array}{l}
w_{1} \in Y, \\
s_{n}=\left(1-\beta_{n}\right) w_{n} \oplus \beta_{n} u_{n}, \\
w_{n+1}=\left(1-\alpha_{n}\right) u_{n} \oplus \alpha_{n} v_{n}, \quad n \geq 1,
\end{array}\right.
$$

where $u_{n} \in F w_{n}$ such that $\rho\left(w_{n}, u_{n}\right)=\mathscr{R}\left(w_{n}, F w_{n}\right)$ and $v_{n}$ $\in F s_{n}$ such that $\rho\left(s_{n}, v_{n}\right)=\mathscr{R}\left(s_{n}, F s_{n}\right)$. The purpose of this work is to prove, under some appropriate conditions, the strong and $\Delta$ convergence results of stationary points for a wider class of multivalued nonexpansive mappings socalled multivalued Suzuki mappings using iterative process (6) in the general setting of 2-uniformly convex hyperbolic spaces. In this way, we improve and extend the corresponding results proved in [21-25].

Now we recall some basic definitions and results, which will be used in the sequel.

Definition 2 [26]. A hyperbolic space is a metric space $(M, \rho)$ endowed with a function $H: M \times M \times[0,1] \longrightarrow M$ such that for all $a, b, z, w \in M$ and $i, s \in[0,1]$, we have

$$
\begin{aligned}
& (H 1) \rho(z, H(a, b, i)) \leq(1-i) \rho(z, a)+i \rho(z, b) \\
& (H 2) \rho(H(a, b, i), H(a, b, j)=|i-j| \rho(a, b) \\
& (H 3) H(a, b, i)=H(b, a, 1-i) \\
& (H 4) \rho(H(a, z, i), H(b, w, i)) \leq(1-i) \rho(a, b)+i \rho(z, w)
\end{aligned}
$$

If $a, b \in M$ and $i \in[0,1]$, we use the notation $(1-i) a \oplus i b$ for $H(a, b, i)$. It follows from $(H 1)$ that

$$
\begin{aligned}
& \rho(a,(1-i) a \oplus i b)=i \rho(a, b), \\
& \rho(b,(1-i) a \oplus i b)=(1-i) \rho(a, b) .
\end{aligned}
$$

The set $\varnothing \neq Y \subseteq M$ is called convex if for any $a, b \in Y$, one has $[a, b]=\{(1-\gamma) a \oplus \gamma b: \gamma \in[0,1]\} \subseteq Y$.

Definition 3. A hyperbolic space $(M, \rho, H)$ is said to be uniformly convex if for every real number $r>0$ and $\epsilon \in(0,2]$, we can choose a $\sigma \in(0,1]$ such that for each $a, b, z \in M$ with $\rho(a, z) \leq r, \rho(b, z) \leq r$, and $\rho(a, b) \geq 2 \epsilon$, one has

$$
\rho\left(\frac{1}{2} a \oplus \frac{1}{2} b\right) \leq(1-\sigma) r
$$

A function $\eta:(0, \infty) \times(0,2] \longrightarrow(0,1]$ providing such $\sigma=\eta(r, \epsilon)$ for given $r \in(0, \infty)$ and $\epsilon \in(0,2]$ is called a modulus of uniform convexity. The function $\eta$ is called monotone provided that it is nonincreasing in $r$ for each fixed $\epsilon$.

Definition 4. Let $(M, \rho)$ be a uniformly convex hyperbolic space. For every $r \in(0, \infty)$ and $\epsilon \in(0,2]$, set

$$
\varphi(r, \epsilon)=\inf \left\{\frac{1}{2} \rho^{2}(a, z)+\frac{1}{2} \rho^{2}(b, z)-\rho^{2}\left(\frac{1}{2} a \oplus \frac{1}{2} b, z\right)\right\},
$$

where the infimum is taken over each $a, b, z \in M$ such that $\rho(a, z) \leq r, \rho(b, z) \leq r$ and $\rho(a, b) \geq r \epsilon$. We say that $(M, \rho)$ is 2-uniformly convex if

$$
c_{M}=\inf \left\{\frac{\varphi(r, \varepsilon)}{r^{2} \varepsilon^{2}}: r \in(0, \infty), \epsilon \in(0,2]\right\}>0 .
$$

Remark 5. Notice that uniformly convex Banach space and CAT(0) spaces as well as $\mathrm{CAT}(\kappa)$ spaces $(\kappa>0$ and diam $(M) \leq\left((\pi / 2-\epsilon) / \kappa^{1 / 2}\right)$ for some $\epsilon \in(0, \pi / 2)$ are 2 -uniformly convex hyperbolic spaces (see $[23,27,28])$.

Definition 6. Let $\left\{w_{n}\right\}$ be any bounded sequence in a complete 2 -uniformly convex hyperbolic space $M$ and $\varnothing \neq$ 
$Y \subseteq M$. The asymptotic radius of $\left\{w_{n}\right\}$ relative to $Y$ is $r(Y$, $\left.\left\{w_{n}\right\}\right)=\inf \left\{\lim \sup _{n \rightarrow \infty} \rho\left(w_{n}, w\right): w \in Y\right\}$. Moreover, the asymptotic center of $\left\{w_{n}\right\}$ relative to $Y$ is the set $\left.A\left(Y, w_{n}\right\}\right)$ $=\left\{w \in Y: \lim \sup _{n \rightarrow \infty} \rho\left(w_{n}, w\right)=r\left(Y, w_{n}\right)\right\}$.

Definition 7. Let $Y$ be a nonempty closed convex subset in a complete 2-uniformly convex hyperbolic space $M$ and $w \epsilon$ $Y$. Let $\left\{w_{n}\right\}$ be any bounded sequence in $M$. We say that $\left\{w_{n}\right\} \Delta$-converges to $w$ if $A\left(Y,\left\{z_{n}\right\}\right)=\{w\}$ for each subsequence $\left\{z_{n}\right\}$ of $\left\{w_{n}\right\}$. In this case, we write $\Delta-\lim _{n \rightarrow \infty} w_{n}=$ $w$ and call $w$ the $\Delta$-lim of $\left\{w_{n}\right\}$.

Now, we collect some basic facts about multivalued Suzuki mappings, which can be found in [29-31].

Proposition 8. Let $Y$ be a nonempty subset of a complete 2uniformly convex hyperbolic space and $F: Y \longrightarrow \mathscr{C}(Y)$.

(i) If $F$ is a Suzuki mapping with a nonempty fixed point set, then $F$ is quasinonexpansive

(ii) If $F$ is a Suzuki mapping, then the following holds:

$$
\rho(a, F b) \leq 3 \rho(a, F a)+\rho(a, b) \quad \text { for all } a, b \in Y \text {. }
$$

The following facts are also needed.

Lemma 9 (see [17]). Let $\alpha_{n}, \beta_{n} \in[0,1)$ be such that $\lim _{n \rightarrow \infty}$ $\beta_{n}=0$ and $\sum \alpha_{n} \beta_{n}=\infty$. Let $\left\{\gamma_{n}\right\}$ be a sequence of nonnegative real numbers such that $\sum \alpha_{n} \beta_{n}\left(1-\beta_{n}\right) \gamma_{n}<\infty$. Then, $\left\{\gamma_{n}\right\}$ has a subsequence which converges to 0 .

The following lemma is a characterization of 2-uniformly convex hyperbolic spaces.

Lemma 10 (see [23]). Let $(M, \rho)$ be a 2 -uniformly convex hyperbolic space. Then,

$\rho^{2}((1-\xi) a \oplus \xi b, p) \leq(1-\xi) \rho^{2}(a, p)+\xi \rho^{2}(b, p)-4 c_{M} \xi(1-\xi) \rho^{2}(a, b)$,

for each $\xi \in[0,1]$ and $a, b, p \in M$.

Lemma 11 (see [23]). Let $Y$ be a nonempty closed convex subset of a2-uniformly convex hyperbolic space $M$ and $F: Y$ $\longrightarrow \mathscr{C}(Y)$ be a Suzuki mapping. Suppose that $\left\{w_{n}\right\}$ is a bounded sequence in $Y$ such that $\lim _{n \rightarrow \infty} \mathscr{R}\left(w_{n}, F w_{n}\right)=0$ and $\left\{\rho\left(w_{n}, e\right)\right\}$ converges for every $e \in \mathcal{S}(F)$, then $\omega_{\omega}\left(w_{n}\right) \subseteq$ $\delta(F)$. Here, $\omega_{\omega}\left(w_{n}\right)=\cup A\left(Y,\left\{z_{n}\right\}\right)$ where the union is taken over all subsequences $\left\{z_{n}\right\}$ of $\left\{w_{n}\right\}$. Furthermore, $\omega_{\omega}\left(w_{n}\right)$ is a singleton.

\section{Convergence Theorems in 2-Uniformly Convex Hyperbolic Spaces}

Throughout the section, $M$ will stand for a complete 2uniformly convex hyperbolic space with monotone modulus of uniform convexity.
The following lemma is crucial.

Lemma 12. Let $Y$ be a nonempty closed convex subset of $M$ and $F: Y \longrightarrow \mathscr{C}(Y)$ be a Suzuki mapping with $\delta(F) \neq \varnothing$. Let $\left\{w_{n}\right\}$ be the sequence defined by (6). Then, $\lim _{n \rightarrow \infty} \rho\left(w_{n}\right.$, q) exists for all $q \in \mathcal{S}(F)$.

Proof. Let $q \in \mathcal{S}(F)$. By Proposition 8(i), we have

$$
\begin{aligned}
\rho\left(w_{n+1}, q\right) \leq & \left(1-\alpha_{n}\right) \rho\left(u_{n}, q\right)+\alpha_{n} \rho\left(v_{n}, q\right) \\
= & \left(1-\alpha_{n}\right) \rho\left(u_{n}, F q\right)+\alpha_{n} \rho\left(v_{n}, F q\right) \\
\leq & \left(1-\alpha_{n}\right) \mathbb{H}\left(F w_{n}, F q\right)+\alpha_{n} \mathbb{H}\left(F s_{n}, F q\right) \\
\leq & \left(1-\alpha_{n}\right) \rho\left(w_{n}, q\right)+\alpha_{n} \rho\left(s_{n}, q\right) \leq\left(1-\alpha_{n}\right) \rho\left(w_{n}, q\right) \\
& +\alpha_{n}\left[\left(1-\beta_{n}\right) \rho\left(w_{n}, q\right)+\beta_{n} \rho\left(u_{n}, q\right)\right] \\
= & \left(1-\alpha_{n}\right) \rho\left(w_{n}, q\right)+\alpha_{n}\left[\left(1-\beta_{n}\right) \rho\left(w_{n}, F q\right)\right. \\
& \left.+\beta_{n} \rho\left(w_{n}, F q\right)\right] \leq\left(1-\alpha_{n}\right) \rho\left(w_{n}, q\right) \\
& +\alpha_{n}\left[\left(1-\beta_{n}\right) \rho\left(w_{n}, q\right)+\beta_{n} \mathbb{H}\left(F w_{n}, F q\right)\right] \\
\leq & \left(1-\alpha_{n}\right) \rho\left(w_{n}, q\right)+\alpha_{n}\left[\left(1-\beta_{n}\right) \rho\left(w_{n}, q\right)\right. \\
& \left.+\beta_{n} \rho\left(w_{n}, q\right)\right]=\left(1-\alpha_{n}\right) \rho\left(w_{n}, q\right) \\
& +\alpha_{n} \rho\left(w_{n}, q\right)=\rho\left(w_{n}, q\right) .
\end{aligned}
$$

Hence, $\left\{\rho\left(w_{n}, q\right)\right\}$ is a nonincreasing sequence, which implies $\lim _{n \rightarrow \infty} \rho\left(w_{n}, q\right)$ exists for every $q \in \delta(F)$.

First, we establish our $\Delta$-convergence theorem.

Theorem 13. Let $Y$ be a nonempty closed convex subset of $M$ and $F: Y \longrightarrow \mathscr{C}(Y)$ be a Suzuki mapping with $\delta(F)) \neq \varnothing$. Let $\alpha_{n}, \beta_{n} \in[a, b] \subset(0,1)$ and $\left\{w_{n}\right\}$ be the sequence defined by (6). Then, $\left\{w_{n}\right\} \Delta$-converges to a stationary point of $F$.

Proof. Fix $q \in \mathcal{S}(F)$. By Lemma 10, we have

$$
\begin{aligned}
\rho^{2}\left(s_{n}, q\right) \leq & \left(1-\beta_{n}\right) \rho^{2}\left(w_{n}, q\right)+\beta_{n} \rho^{2}\left(u_{n}, q\right) \\
& -4 c_{M} \beta_{n}\left(1-\beta_{n}\right) \rho^{2}\left(w_{n}, u_{n}\right) \\
\leq & \left(1-\beta_{n}\right) \rho^{2}\left(w_{n}, q\right)+\beta_{n} \mathbb{H}^{2}\left(F w_{n}, F q\right) \\
& -4 c_{M} \beta_{n}\left(1-\beta_{n}\right) \rho^{2}\left(w_{n}, u_{n}\right) \\
\leq & \left(1-\beta_{n}\right) \rho^{2}\left(w_{n}, q\right)+\beta_{n} \rho^{2}\left(w_{n}, q\right) \\
& -4 c_{M} \beta_{n}\left(1-\beta_{n}\right) \rho^{2}\left(w_{n}, u_{n}\right) \\
\leq & \rho^{2}\left(w_{n}, q\right)-4 c_{M} \beta_{n}\left(1-\beta_{n}\right) \rho^{2}\left(w_{n}, u_{n}\right) .
\end{aligned}
$$

Thus,

$$
\begin{aligned}
\rho^{2}\left(w_{n+1}, q\right) \leq & \left(1-\alpha_{n}\right) \rho^{2}\left(u_{n}, q\right)+\alpha_{n} \rho^{2}\left(v_{n}, q\right) \\
& -4 c_{M} \alpha_{n}\left(1-\alpha_{n}\right) \rho^{2}\left(u_{n}, v_{n}\right) \\
\leq & \left(1-\alpha_{n}\right) \mathbb{H}^{2}\left(F w_{n}, F q\right)+\alpha_{n} \mathbb{H}^{2}\left(F s_{n}, F q\right) \\
& -4 c_{M} \alpha_{n}\left(1-\alpha_{n}\right) \rho^{2}\left(u_{n}, v_{n}\right) \\
\leq & \left(1-\alpha_{n}\right) \rho^{2}\left(w_{n}, q\right)+\alpha_{n} \rho^{2}\left(s_{n}, q\right) \\
& -4 c_{M} \alpha_{n}\left(1-\alpha_{n}\right) \rho^{2}\left(u_{n}, v_{n},\right) \\
\leq & \left(1-\alpha_{n}\right) \rho^{2}\left(w_{n}, q\right)+\alpha_{n} \rho^{2}\left(s_{n}, q\right) \\
\leq & \rho^{2}\left(w_{n}, q\right)-4 c_{M} \alpha_{n} \beta_{n}\left(1-\beta_{n}\right) \rho^{2}\left(w_{n}, u_{n}\right) .
\end{aligned}
$$


Since $c_{M}>0$, it follows that

$\sum_{n=1}^{\infty} a^{2}(1-b) \rho^{2}\left(w_{n}, u_{n}\right) \leq \sum_{n=1}^{\infty} \alpha_{n} \beta_{n}\left(1-\beta_{n}\right) \rho^{2}\left(w_{n}, u_{n}\right)<\infty$.

Thus, $\lim _{n \rightarrow \infty} \rho^{2}\left(w_{n}, u_{n}\right)=0$ and hence

$$
\lim _{n \rightarrow \infty} \mathscr{R}\left(w_{n}, F w_{n}\right)=\lim _{n \rightarrow \infty} \rho\left(w_{n}, u_{n}\right)=0 .
$$

By Lemma 12, $\left\{\rho\left(w_{n}, e\right)\right\}$ converges for all $e \in \mathcal{S}(F)$. By Lemma $11, \omega_{\omega}\left(w_{n}\right)$ is a singleton and is contained in $\mathcal{S}(F)$. This shows that $\left\{w_{n}\right\} \Delta$-converges to a point of $\mathcal{S}(F)$.

Definition 14 (see [21]). Let $Y$ be a nonempty subset of $M$ and $F: Y \longrightarrow \mathscr{C}(Y) . F$ is said to satisfy condition $J$ if there is a nondecreasing function $\lambda:[0, \infty) \longrightarrow[0, \infty)$ with the properties $\lambda(0)=0, \lambda(r)>0$ for $r>0$ and $\mathscr{R}(a, F a) \geq \lambda(\rho$ $(a, \mathcal{S}(F)))$ for all $a \in Y$. $F$ is called semicompact if for each sequence $\left\{w_{n}\right\}$ in $Y$ satisfying $\lim _{n \rightarrow \infty} \mathscr{R}\left(w_{n}, F w_{n}\right)=0$, one can find a strongly convergent subsequence $\left\{w_{n_{k}}\right\}$ of $\left\{w_{n}\right\}$. Moreover, a sequence $\left\{w_{n}\right\}$ in $M$ is called a Fejér monotone with respect to $Y$ provided that $\rho\left(w_{n+1}, z\right) \leq \rho\left(w_{n}, z\right)$, for each $z \in Y$ and $n \geq 1$.

The following facts are in [31].

Proposition 15. Let $Y$ be a nonempty closed subset of $M$ and $\left\{w_{n}\right\}$ be a Fejér monotone sequence with respect to $Y$. Then, $\left\{w_{n}\right\}$ converges strongly to a point of $Y$ if and only if $\lim _{n \rightarrow \infty} \rho\left(w_{n}, Y\right)=0$. of $F$.

The following theorem is based on the semicompactness

Theorem 16. Let $Y$ be a nonempty closed convex subset of $M$ and $F: Y \longrightarrow \mathscr{C}(Y)$ be a Suzuki mapping with $S(F) \neq \varnothing$. Suppose $\alpha_{n}, \beta_{n} \in[0,1)$ be such that $\sum \alpha_{n} \beta_{n}=\infty$ and $\beta_{n} \longrightarrow$ 0 . If $F$ is semicompact, then $\left\{w_{n}\right\}$ generated by (6) converges strongly to a stationary point of F.

Proof. In view of (16),

$$
\sum_{n=1}^{\infty} \alpha_{n} \beta_{n}\left(1-\beta_{n}\right) \rho^{2}\left(w_{n}, u_{n}\right)<\infty
$$

By Lemma 9, there exists subsequences, namely, $\left\{w_{n_{t}}\right\}$ and $\left\{u_{n_{t}}\right\}$ of $\left\{w_{n}\right\}$ and $\left\{u_{n}\right\}$, respectively, such that $\lim _{t \rightarrow \infty}$ $\rho^{2}\left(w_{n_{t}}, u_{n_{t}}\right)=0$. Hence,

$$
\lim _{t \rightarrow \infty} \mathscr{R}\left(w_{n_{t}}, F w_{n_{t}}\right)=\lim _{t \rightarrow \infty} \rho\left(w_{n_{t}}, u_{n_{t}}\right)=0
$$

By the semicompactness of the mapping $F$, one can find a strongly convergent subsequence $\left\{w_{n_{t}}\right\}$ of $\left\{w_{n}\right\}$ with the strong limit, say $z$. We shall prove that $z \in \mathcal{S}(F)$. By Proposition 8(ii), we have

$$
\begin{aligned}
\rho(z, F z) & \leq \rho\left(z, w_{n_{t}}\right)+\rho\left(w_{n_{t}}, F z\right) \\
& \leq \rho\left(w_{n_{t}}, z\right)+3 \rho\left(w_{n_{t}}, F w_{n_{t}}\right)+\rho\left(w_{n_{t}}, z\right) \\
& =2 \rho\left(w_{n_{t}}, z\right)+3 \rho\left(w_{n_{t}}, F w_{n_{t}}\right) \longrightarrow 0 .
\end{aligned}
$$

Hence, $z \in F z$. By Proposition 8(i),

$$
\mathbb{H}\left(F w_{n_{t}}, F z\right) \leq \rho\left(w_{n_{t}}, z\right) \longrightarrow 0 .
$$

Now, we let $v \in F z$ and choose $y_{n_{t}} \in F w_{n_{t}}$ such that $\rho(v$, $\left.y_{n_{t}}\right)=\rho\left(v, F w_{n_{t}}\right)$. From (19) and (21), we have

$$
\begin{aligned}
\rho(z, v) & \leq \rho\left(z, w_{n_{t}}\right)+\rho\left(w_{n_{t}}, y_{n_{t}}\right)+\rho\left(y_{n_{t}}, v\right) \\
& =\rho\left(z, w_{n_{t}}\right)+\rho\left(w_{n_{t}}, y_{n_{t}}\right)+\rho\left(v, F w_{n_{t}}\right) \\
& \leq \rho\left(z, w_{n_{t}}\right)+\mathscr{R}\left(w_{n_{t}}, F w_{n_{t}}\right)+\mathbb{H}\left(F w_{n_{t}}, F z\right) \longrightarrow 0 .
\end{aligned}
$$

Hence, $v=z$ for all $v \in F z$, that is, $\{z\}=F z$. Therefore, $z$ $\in \mathcal{S}(F)$. By Lemma $12, \lim _{n \rightarrow \infty} \rho\left(w_{n}, z\right)$ exists. Hence, $z$ is the strong limit of $\left\{w_{n}\right\}$.

Example 17. Let $Y$ and $F$ be as in Example 1. Then, $F$ is a Suzuki mapping with $S(F)=\{1\}$. Semicompactness of $F$ follows from the compactness of $Y$. For each $n \geq 1$, we let $\alpha_{n}=$ $1 / 2$ and $\beta_{n}=1 / n$. The, $\lim _{n \rightarrow \infty} \beta_{n}=0$ and $\sum \alpha_{n} \beta_{n}=\infty$. Hence, by Theorem 16, the sequence of the modified Agarwal-O'Regan-Sahu iteration defined by (6) converges strongly to 1 . However, we cannot directly apply any result in $[22,24,25]$ because, in this situation, $F$ is not nonexpansive.

The following theorem requires condition $J$.

Theorem 18. Let $Y$ be a nonempty closed convex subset of $M$ and $F: Y \longrightarrow \mathscr{C}(Y)$ be a Suzuki mapping with $\delta(F) \neq \varnothing$. Suppose $\alpha_{n}, \beta_{n} \in[a, b] \subset(0,1)$. If F satisfies condition $J$, then $\left\{w_{n}\right\}$ generated by (6) converges strongly to a stationary point of $F$.

Proof. From (17), we have

$$
\lim _{n \rightarrow \infty} \mathscr{R}\left(w_{n}, F w_{n}\right)=0
$$

Since $F$ satisfies condition $J$, we have

$$
\mathscr{R}\left(w_{n}, F w_{n}\right) \geq \lambda\left(\rho\left(w_{n}, \mathcal{S}(F)\right)\right)
$$

So, from (23), we get

$$
\lim _{n \rightarrow \infty} \lambda\left(\rho\left(w_{n}, \mathcal{S}(F)\right)=0\right.
$$


Since the function $\lambda$ is nondecreasing and $\lambda(0)=0$ and $\lambda(r)>0$ for every $r>0$. Hence,

$$
\lim _{n \rightarrow \infty} \rho\left(w_{n}, \mathcal{S}(F)\right)=0
$$

Closeness of $\mathcal{S}(F)$ follows from the quasinonexpansiveness of $F$. In the view of Lemma 12, we have $\left\{w_{n}\right\}$ which is a Fejér monotone with respect to $\delta(F)$. By Proposition 15, $\left\{w_{n}\right\}$ converges strongly to an element of $\mathcal{S}(F)$.

Example 19. Let $Y$ and $F$ be as in Example 1. Then, $F$ is a Suzuki mapping with $\delta(F)=\{1\}$ and satisfies the condition $J$. For each $n \geq 1$, we let $\alpha_{n}=\beta_{n}=1 / 3$. Then, by Theorem 18, the sequence of modified Agarwal-O'Regan-Sahu iteration defined by (6) converges strongly to 1 . However, we cannot directly apply any result in $[22,24,25]$ because, in this situation, $F$ is not nonexpansive.

\section{Conclusions}

Under some appropriate conditions, we have proven that the sequence of the modified Agarwal-O'Regan-Sahu iterative process defined by (6) converges to a stationary point of a multivalued Suzuki mapping. We have used a 2-uniformly convex hyperbolic space, which is more general than the uniformly convex Banach spaces, CAT(0) spaces, and some CAT $(\kappa)$ spaces. Moreover, the class of multivalued Suzuki mappings properly includes the class of multivalued nonexpansive mappings as shown by Example 1. Our iterative process is independent of but better than the Ishikawa iterative process. Hence, our presented results extend and improve the corresponding results in [21-25].

\section{Data Availability}

No data were used to support this study.

\section{Conflicts of Interest}

The authors declare that they have no conflicts of interest.

\section{Authors' Contributions}

All authors contributed equally and significantly in writing this article. All authors read and approved the final manuscript.

\section{Acknowledgments}

The authors are grateful to the Spanish Government for Grant RTI2018-094336-B-I00 (MCIU/AEI/EU FEDER) and to the Basque Government for Grant IT1207-19.

\section{References}

[1] A. Abkar and M. Eslamian, "A fixed point theorem for generalized nonexpansive multivalued mappings," Fixed Point Theory, vol. 12, pp. 241-246, 2011.
[2] K. Ullah, F. Ayaz, and J. Ahmad, "Some convergence results of M iterative process in Banach spaces," Asian-European Journal of Mathematics, article 2150017, 2019.

[3] T. Abdeljawad, K. Ullah, and J. Ahmad, "On Picard-Krasnoselskii Hybrid Iteration Process in Banach Spaces," Journal of Mathematics, vol. 2020, Article ID 2150748, 5 pages, 2020.

[4] T. Abdeljawad, K. Ullah, and J. Ahmad, "Iterative Algorithm for Mappings Satisfying $\left(B_{\gamma, \mu}\right)$ Condition," Journal of Function Spaces, vol. 2020, Article ID 3492549, 7 pages, 2020.

[5] K. Ullah, J. Ahmad, and M. de la Sen, "On generalized nonexpansive maps in Banach spaces," Computation, vol. 8, no. 3, p. $61,2020$.

[6] T. Abdeljawad, K. Ullah, J. Ahmad, and N. Mlaiki, "Iterative approximations for a class of generalized nonexpansive operators in Banach spaces," Discrete Dynamics in Nature and Society, vol. 2020, Article ID 4627260, 6 pages, 2020.

[7] K. Ullah, K. Iqbal, and M. Arshad, "Some convergence results using $K$ iteration process in CAT(0) spaces," Fixed Point Theory and Applications, vol. 2018, no. 1, Article ID 11, 2018.

[8] B. Panyanak, "Stationary points of lower semicontinuous multifunctions," Journal of Fixed Point Theory and Applications, vol. 22, no. 2, article 43, 2020.

[9] J. P. Aubin and J. Siegel, "Fixed points and stationary points of dissipative multivalued maps," Proceedings of American Mathematical Society, vol. 78, no. 3, pp. 391-398, 1980.

[10] L. Chen, L. Gao, and D. Chen, "Fixed point theorems of mean nonexpansive set-valued mappings in Banach spaces," Journal of Fixed Point Theory and Applications, vol. 19, no. 3, pp. 2129-2143, 2017.

[11] R. Espinola, M. Hosseini, and K. Nourouzi, "On stationary points of nonexpansive set-valued mappings," Fixed Point Theory and Applications, vol. 2015, no. 1, Article ID 236, 2015.

[12] M. Hosseini, K. Nourouzi, and D. O'Regan, “Stationary points of set-valued contractive and nonexpansive mappings on ultrametric spaces," Fixed Point Theory, vol. 19, no. 2, pp. 587-594, 2018.

[13] B. Panyanak, "Endpoints of multivalued nonexpansive mappings in geodesic spaces," Fixed Point Theory and Applications, vol. 2015, no. 1, Article ID 147, 2015.

[14] S. Reich, "Fixed points of contractive functions," Bollettino dell'Unione Matematica Italiana, vol. 5, pp. 26-42, 1972.

[15] S. Saejung, "Remarks on endpoints of multivalued mappings in geodesic spaces," Fixed Point Theory and Applications, vol. 2016, no. 1, Article ID 52, 2016.

[16] K. P. R. Sastry and G. V. R. Babu, "Convergence of Ishikawa iterates for a multivalued mapping with a fixed point," Czechoslovak Mathematical Journal, vol. 55, no. 4, pp. 817-826, 2005.

[17] B. Panyanak, "Mann and Ishikawa iterative processes for multivalued mappings in Banach spaces," Computers \& Mathematcs with Applications, vol. 54, no. 6, pp. 872-877, 2007.

[18] Y. Song and H. Wang, "Erratum to "Mann and Ishikawa iterative processes for multivalued mappings in Banach spaces", Computers \& Mathematics with Applications, vol. 55, no. 12, pp. 2999-3002, 2008.

[19] N. Shahzad and H. Zegeye, "On Mann and Ishikawa iteration schemes for multivalued maps in Banach spaces," Nonlinear Analysis, vol. 71, no. 3-4, pp. 838-844, 2009.

[20] Y. Song and Y. J. Cho, "Some notes on Ishikawa iteration for multi-valued mappings," Bulletin of the Korean Mathematical Society, vol. 48, no. 3, pp. 575-584, 2011. 
[21] B. Panyanak, "Approximating endpoints of multi-valued nonexpansive mappings in Banach spaces," Journal of Fixed Point Theory and Applications, vol. 20, no. 2, article 77, 2018.

[22] K. Ullah, M. S. U. Khan, N. Muhammad, and J. Ahmad, "Approximation of endpoints for multivalued nonexpansive mappings in geodesic spaces," Asian-European Journal of Mathematics, article 2050141, 2019.

[23] T. Laokul and B. Panyanak, "A generalization of the $(\mathrm{CN})$ inequality and its applications," Carpathian Journal of Mathematics, vol. 36, no. 1, pp. 81-90, 2020.

[24] T. Abdeljawad, K. Ullah, J. Ahmad, and N. Mlaiki, "Iterative approximation of endpoints for multivalued mappings in Banach spaces," Journal of Function Spaces, vol. 2020, Article ID 2179059, 5 pages, 2020.

[25] K. Ullah, J. Ahmad, and N. Muhammad, "Approximation of endpoints for multi-valued mappings in metric spaces," Journal of Linear and Toplogical Algebra, vol. 9, no. 2, pp. 129$137,2020$.

[26] L. Leustean, "A quadratic rate of asymptotic regularity for CAT(0) spaces," Journal of Mathematical Analysis and Applications, vol. 325, no. 1, pp. 386-399, 2007.

[27] M. A. Khamsi and A. R. Khan, "Inequalities in metric spaces with applications," Nonlinear Analysis, vol. 74 , no. 12, pp. 4036-4045, 2011.

[28] H. K. Xu, "Inequalities in Banach spaces with applications," Nonlinear Analysis, vol. 16, no. 12, pp. 1127-1138, 1991.

[29] R. Espinola, P. Lorenzo, and A. Nicolae, "Fixed points, selections and common fixed points for nonexpansive-type mappings," Journal of Mathematical Analysis and Applications, vol. 382, no. 2, pp. 503-515, 2011.

[30] T. Suzuki, "Fixed point theorems and convergence theorems for some generalized nonexpansive mappings," Journal of Mathematical Analysis and Applications, vol. 340, no. 2, pp. 1088-1095, 2008.

[31] P. Chuadchawna, A. Farajzadeh, and A. Kaewcharoen, "Convergence theorems and approximating endpoints for multivalued Suzuki mappings in hyperbolic spaces," Journal of Computational Analysis and Applications, vol. 28, pp. 903916, 2020. 